\title{
Newer application of Oncothermia to non-malignant diseases such as Dupuytren's contracture of the hand and chronic lower back pain lasting more than 4 weeks
}

\author{
Agnes Mate, MD, \\ PhD student at Pecs University, Health Science Faculty \\ Istvan Molnar MD, \\ $\mathrm{PhD}$ student at Pecs University, Health Science Faculty \\ Henrik Szőke MD, PhD, \\ CAM Division, Health Science Dept., Pecs University, Pecs, Hungary \\ Gabriella Hegyi, MD, MSc., PhD. \\ Prof. \& Head of CAM Division, Health Science Dept., Pécs University, Pécs, Hungary \\ (Correspondence: Gabriella Hegyi MD, MSc, PhD. Address: 1196. Budapest, Petofi u.79, \\ Hungary. Tel: 00-36-309225347; Fax: 00-36-12813035; E-mail: drhegyi@hu.inter.net)
}

(Received: February 2, 2017; Accepted with Revisions: June 22, 2017)

\begin{abstract}
:
Oncothermia (OTM) is based on electromagnetic interactions with the living matter. Its nano-targeting approach establishes a new paradigm. OTM could be applied not only to treat malignancies but it is feasible to apply for other diseases too. OTH is well proven method from the laboratory level to the clinical applications, promoting the natural processes, re-establishing of the synchronization between the cells. This approach allows therapy of some nonmalignant diseases too. Our objective is to use OTM for the irregularities of tissue-growth and for pain syndromes as well. OTM uses precisely tuned radiofrequency $(\mathrm{RF})$ current flowing through the treated part of the body. We made extensive study, proving in details the applicability of the OTM for Dupuytren's contracture and chronic low back pain, in conjunction with permanent acupuncture method, a modified process of traditional Chinese Medicine (TCM). The synergy of the ancient and the state-of-art medical knowledges is the basic method of the present research. Our results with application of OTM for chronic low back pain and Dupuytren's contracture shows definite improvements of the patients involved in the study. We measured the success with the Visual Analog Scale (VAS), with anecdotic quality of life (QoL), and the shortening of the of-work period due to the disease. We measured that VAS decreased significantly, the hand contracture became less rigid and less contracted. The QoL of patients improved and their complaints which blocked their regular activity has significantly reduced. OTM method was safe \& no adverse effects were observed. Recognition of the distortions in the healthy tissue have some common principles and possibilities in TCM and OTM: both the methods recognize the loss of complexity of the living organization. Data of our
\end{abstract}


recent study verified the relevant end-points of our present study: the safety, the quality of life (QoL), the prolonged painless status of the patient.

Keywords: Physiotherapy, Oncothermia, Dupuytren's contracture, Non-specific low back pain

\section{INTRODUCTION}

Oncothermia (OTM) is a kind of hyperthermia method intended to use of malignant diseases, [1]. OTM is based on electromagnetic interactions with the living matter, selecting the malignant cells as targets, [2]. The membrane structure of malignant cells absorbs the provided electromagnetic energy, and controlled signal pathways are excited. This kind of nano-targeting approach establishes a new paradigm, [3]; which was successfully applied for various malignancies [4], which could be applied not only in case of malignancies but other diseases too. When the tissue is not malignant, but partly could be selected by the radiofrequency (RF) current OTM does effect to repair the irregularities as proliferation of non-malignant tissues. [5]. OTM method is well proven from the laboratory level to the clinical applications, [6]. It ignites the natural processes supporting the homeostasis of the system, re-establishing the communication harmony between the cells. This is the reason of our present applications to treat some nonmalignant diseases.

OTM was successfully applied previously for Lyme-disease, [7] for low-back pain, [8] for Peyronie disease, [9] and for Dupuytren's contracture, [10] too. The number of patients was a small one in these studies above. Therefore, we made a more extensive study, proving in details the applicability of the OTM for two of these diseases, the Dupuytren's contracture and the chronic low back pain. Dupuytren's contracture: (also known as Morbus Dupuytren's, or Dupuytren's disease and slang terms "Viking disease" or "Celtic hand"), is a fixed flexion contracture of the hand. It is named after Baron Guillaume Dupuytren, the surgeon who described an operation to correct the affliction in the Lancet in 1831. The Dupuytren's contracture is a palmar fibromatosis, where the fingers bend towards the palm and cannot be fully extended (straightened). The ring finger and little finger are most commonly affected. The middle finger may be affected in advanced cases, but the index finger and the thumb are more rarely involved. It is an inherited proliferative connective tissue disorder that involves the hand's palmar fascia. Dupuytren's contracture progresses slowly and is often accompanied by some aching and itching. In patients with this condition, the palmar fascia thickens and shortens so that the tendons connected to the fingers cannot move freely. Incidence increases after age 40; at this age, men are affected more often than women. Beyond age of 80 the gender distribution is approx. even. Operation can improve the progressive situation in stage N0, N1. (N0, N1, N2 are the most common known stages of disease sorted according its development. N0: starting bending of finger, N1: more improvement fibromatosis, bending is significantly much more without extension ability, N2: no extension of finger anymore, serious fibromatosis) they are and other alternative therapies can be tried, like Quercetin, Bromelain, DMSO (Dimethyl Sulfoxide), MSM (Methylsulfonylmethane) Acetylcarnitin HCl, PABA (Para-amino-bensoic-acid), Nattokinase, Vitamin E Copper, Vitamin C, massage. The palmar fascia becomes hyperplastic and contracts by time to time. 
2. Non-specific or aspecific (according to European rheumatologic classification, we sort any type of low back pain which is not being verified as disc herniation, discopathy, protrusion of disc, originated from tumors, osteoporosis ). Chronic low back pain: (LBP), also known as lower back pain or lumbago, is a common disorder involving the muscles and bones of the back. Duration of the pain may classify low back pain as acute (pain lasting less than six weeks), subchronic (6 to 12 weeks), or chronic (more than 12 weeks). The condition may be further classified by the underlying cause as either mechanical, nonmechanical, or referred pain. In most episodes of low back pain, a specific cause is not identified or even looked for, with the pain believed to be due to mechanical problems such as muscle or joint strain. If the pain does not go away with conservative treatment or if it is accompanied by "red flags" such as unexplained weight loss, fever, or significant problems with feeling or movement, further testing may be needed to look for a serious underlying problem. In most cases, imaging tools such as X-ray computed tomography are not useful and carry their risks. Damaged intervertebral discs cause some low back pain, and the straight leg raise test is helpful to identify this cause. In those with chronic pain, the pain processing system may malfunction, causing large amounts of pain in response to non-serious events. Despite this, the use of imaging in low back pain has increased. The treatment of acute non-specific low back pain is typical with conservative measures: as the use of simple pain medications and the continuation of as much normal activity as the pain allows without bed rest. Chronic non-specific lower back pain needs more management for relieving permanent pain.

Our objective performs an extended study of the OTM method for the Dupuytren's contracture (DuCo) and the chronic low back pain (cLBP), proving its support to the healthy homeostatic equilibrium, and its effective recognition of the deviations from the complex harmony, and treat selectively for improvement of regular functions.

\section{MATERIALS \& METHODS}

We applied OTM with a specialized device called "Booster" (Oncotherm GmbH, Troisdorf Germany). The OTM methods are generally devoted for primer and metastatic malignancies, but Booster was developed for physiotherapies, using the effects and knowledge, which was collected for two decades in oncology. It is devoted to be applied in rheumatology, sports medicine, dermatology and analgesic therapy. The equipment is a table-top device, having precise tuning of the patient, fitting to the actual electric impedance of the targeted body-part. The maximal power is $80 \mathrm{~W}$ working with $6.78 \mathrm{MHz}$ RF-frequency. We applied this equipment in complementary treatment with a modified acupuncture in Traditional Chinese Medicine (TCM) Our special acupuncture method [11] uses permanent triggering of the chosen acupunctural point even for days without taking care on the needle during in this long period of time. This permanent acupuncture method (PAM) well fits to the complementary applications like the treatment with Booster. The treatment was made for patients eligible for inclusion and exclusion criteria of the study.

The study for selected diseases was performed according to Helsinki Declaration and local ethical approval. Rheumatologist outpatient's clinic made a patient selection. The period of study was 2012-2015. 
Number of studied $n=76$ patients with DuCo, and $n=155$ patients with cLBP. The flow-chart of the present study is shown in fig. 1 .

The inclusion criteria for Dupuytren's contracture (DuCo) were

- diagnosed in Dupuytren's contracture

- Age

- Stages N0, N1, N2 (above explained)

- Physical status

- Blood --- pressure ---- rate---- PO2

- Written consent

The exclusion criteria were for patients, which were not fit to inclusion criteria.

The method of study was to investigate whether progression of the disease could be stopped without side-effect together with an elevation of QoL of patients.

According to the above criteria, we selected 76 patients suffering from Dupuytren's contracture in different stages. Some typical disease shown on fig 1.

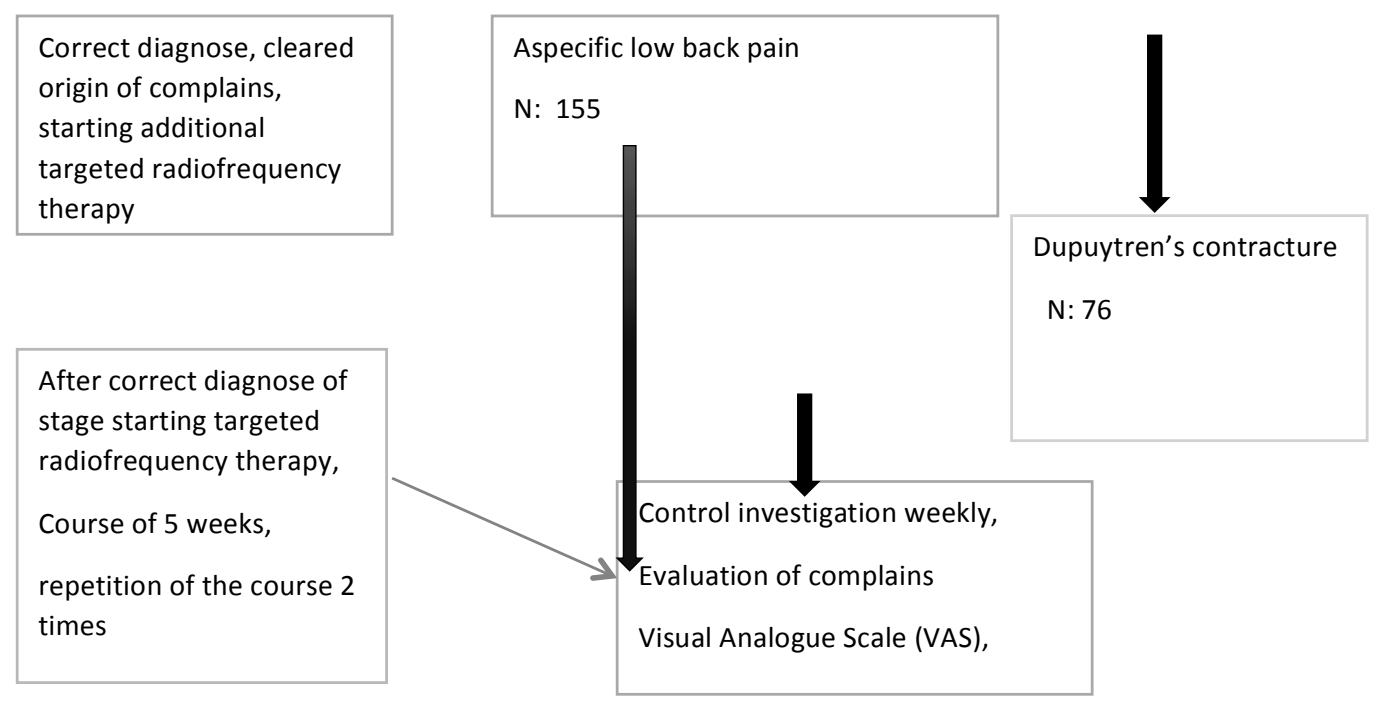

Fig.1. The flow-chart of the present study. 

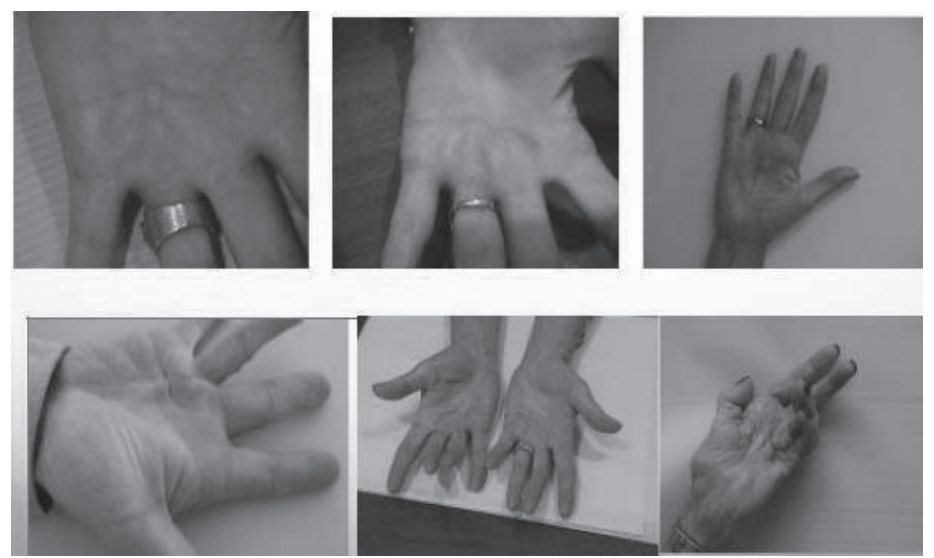

Fig. 2. Dupuytren's contracture N0, N1, N2 stages

The selected patients in all stages received two times/week physiotherapy with the Booster using, $30 \mathrm{~W}, 25$ minutes, one course is four weeks (eight sessions), after $1 / 2$ years it was repeated three times. Additional therapies were applied for Dupuytren's cases: Quercetin, Bromelain, DMSO, MSM, Acetylcarnitin HCl, PABA, Nattokinase, Vitamin E (1940) Copper, Vitamin C, conventional continuous massage therapy. The primer outcome measurement was "top of plate test", (see fig. 3.), a painless extension of palm. Second outcome measurements were QoL, VAS of patients.

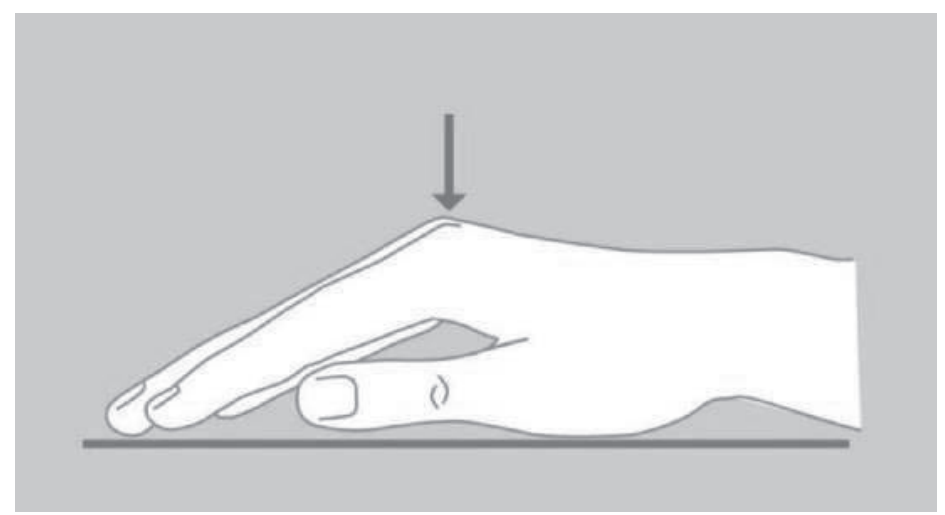

Fig.3. Table-top test. Patient places his hand on a flat surface (e.g. table). If the hand lies completely flat on the table, the test is considered negative. If the hand cannot be placed completely flat on the table, but there is a space between the table and a part of the hand as big as the diameter of a ballpoint pen, the test is considered positive, treatment may be indicated. Additionally, finger joints may become fixed and rigid.

Questionnaire on pain and QoL was filled with all sessions during a physical examination. Visual Analog Scale (VAS), and also the "table-top test" were registered during all period of treatments. 
In case of chronic low back pain (cLBP) the method of study was also to investigate whether progression of the disease could be stopped without side-effect together with an elevation of QoL of patients.

The inclusion criteria for chronic low-back pain were

- diagnosed in chronic low-back pain (cLBP), low back pain lasting more than four weeks

- Age 20-75

- Stage

- Physical status

- Blood -pressure rate- (normal) PO2,

- Written consent

The exclusion criteria were for patients which

- did not fit to inclusion criteria

- have ruptured disc herniation,

- caudal syndrome, (with serious vegetative neurological symptom- as missing reflex of defecation, urination, which needs immediately neurosurgical intervention)

- malignancy (bone metastasis from lung, breast, prostate, thyroid, among others),

- infections (osteomyelitis; abscess).

The patients eligible for criteria received a treatment with Booster 2-times/week, $30 \mathrm{~W}, 25$ minutes, one course is four weeks ( 8 sessions), after $1 / 2$ years it was repeated three times. (example of the treatment is shown on Fig.4.)

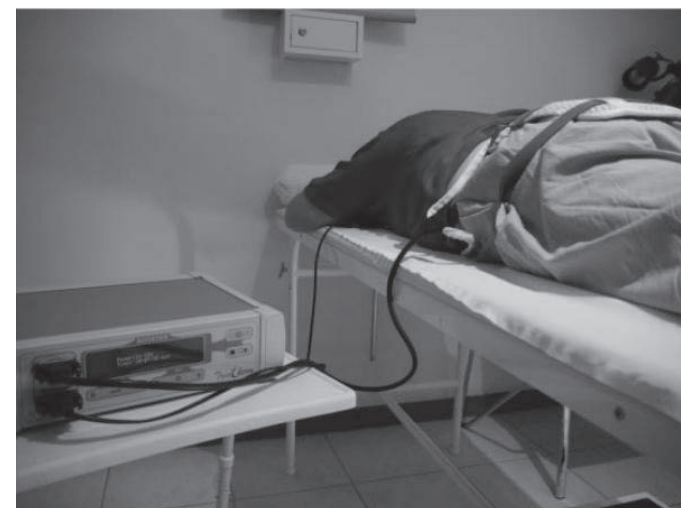

Figure 4a

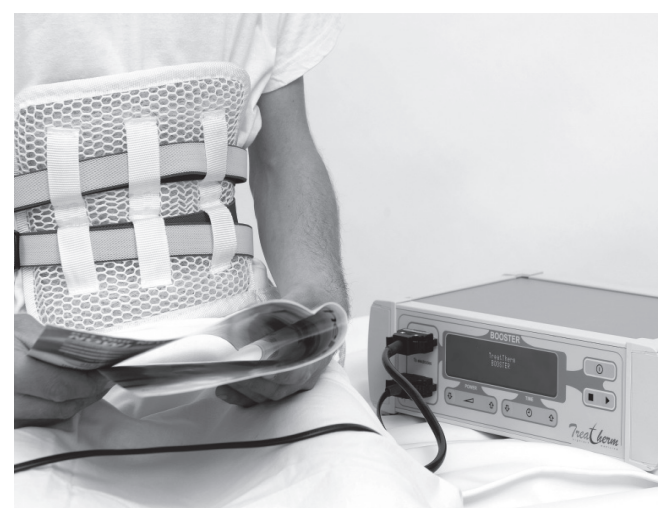

Figure $4 b$ 


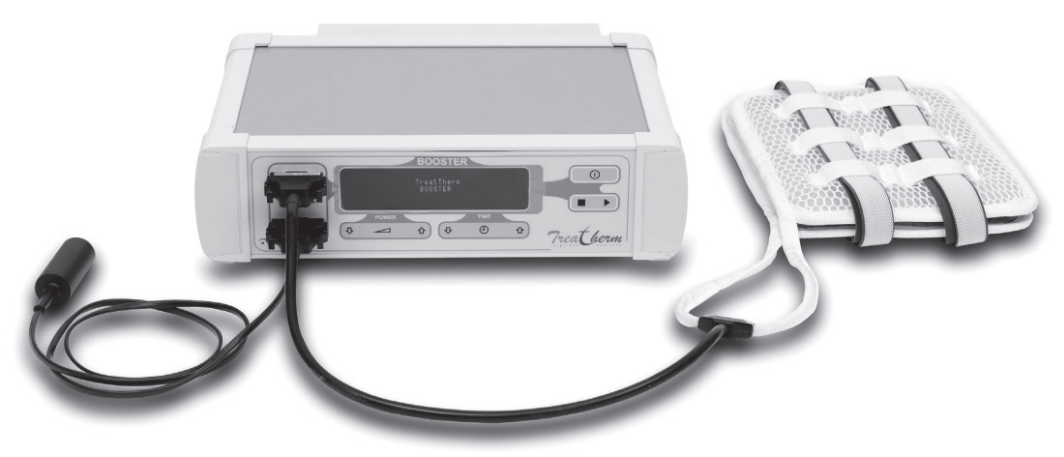

Figure 4c Fig 4. Low back pain treatment in chronic pain

The Booster electrode is applied to the painful local area (back) of the patient. Additionally, all patients received mesotherapy (neuraltherapy by local anaesthetic Procaine 0,5 \%, 2-3 ml /session and acupuncture (permanent monofilament insertion one time/month according to TCM protocol and protocol of Neuraltherapy according to Hunecke). Example of the investigation is shown on Fig. 5.

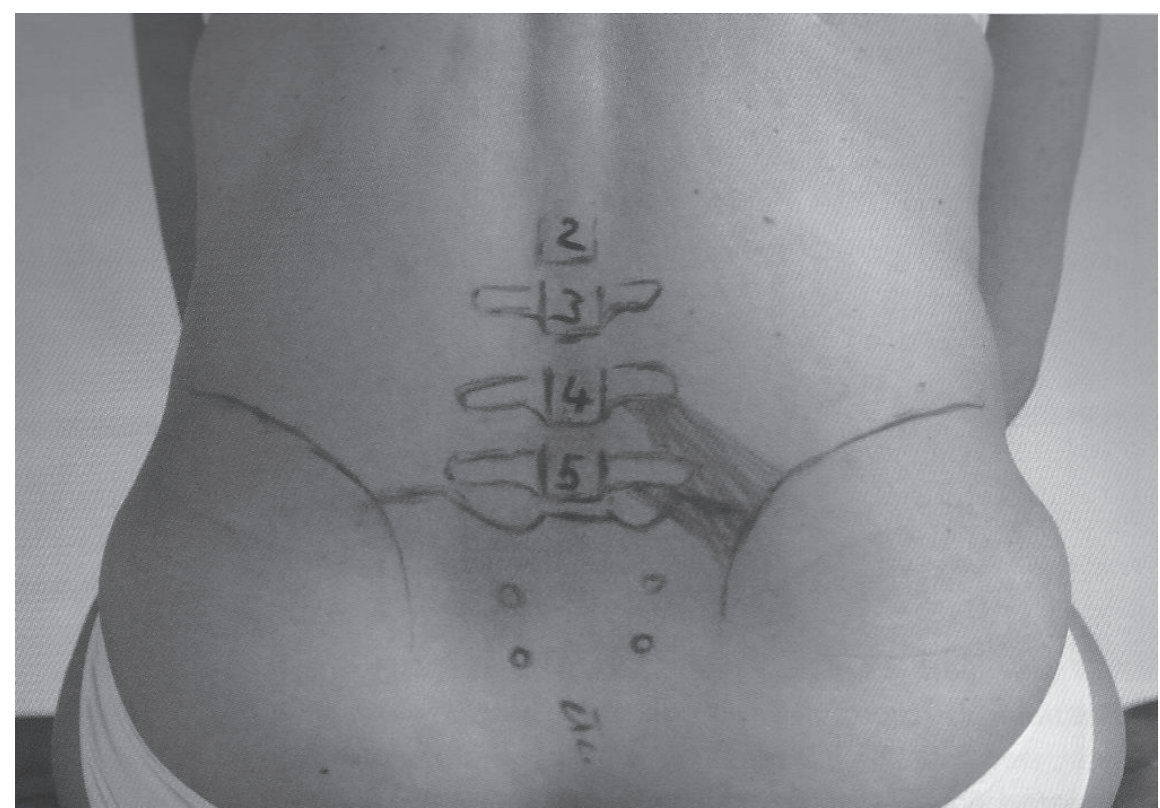

Figure 5a. Anatomical orientation of lumbar-sacral region, spine, muscles and tendons 


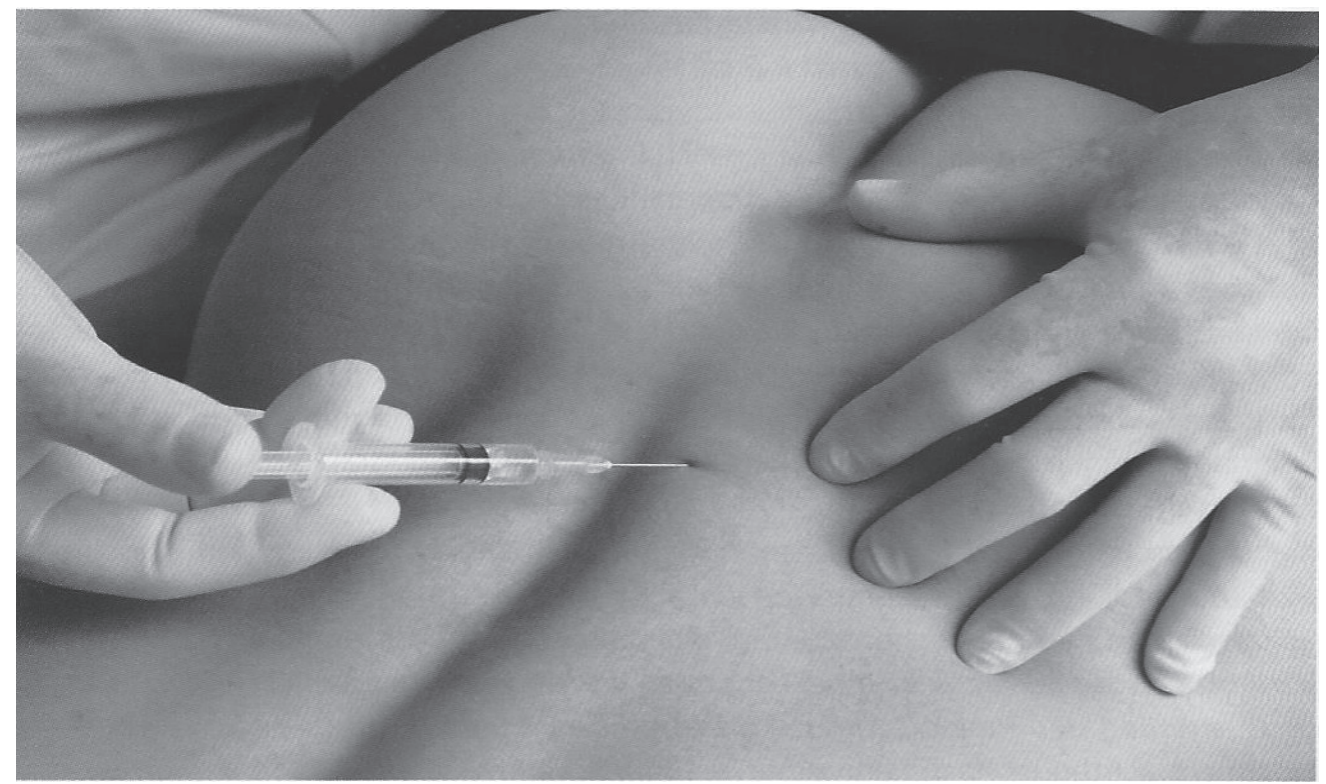

Figure $5 b$
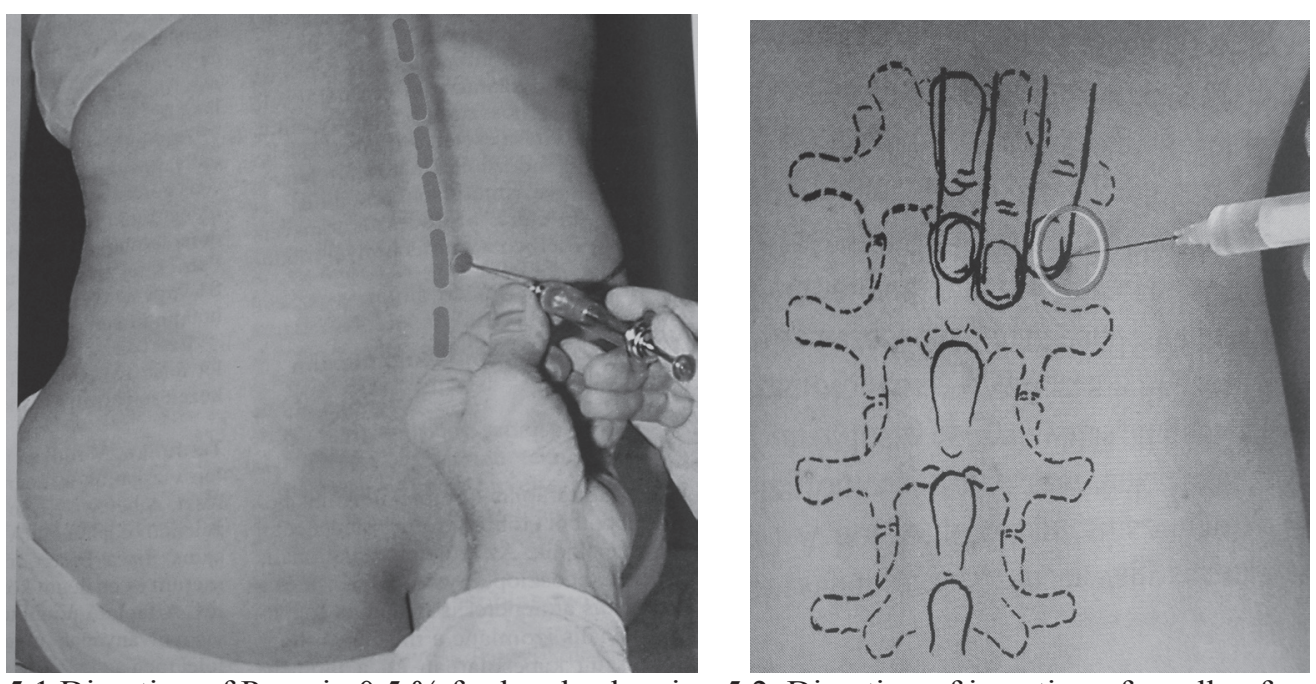

5.1 Direction of Procain 0,5 \% for low back pain 5.2. Direction of insertion of needle of neuraltherapy 


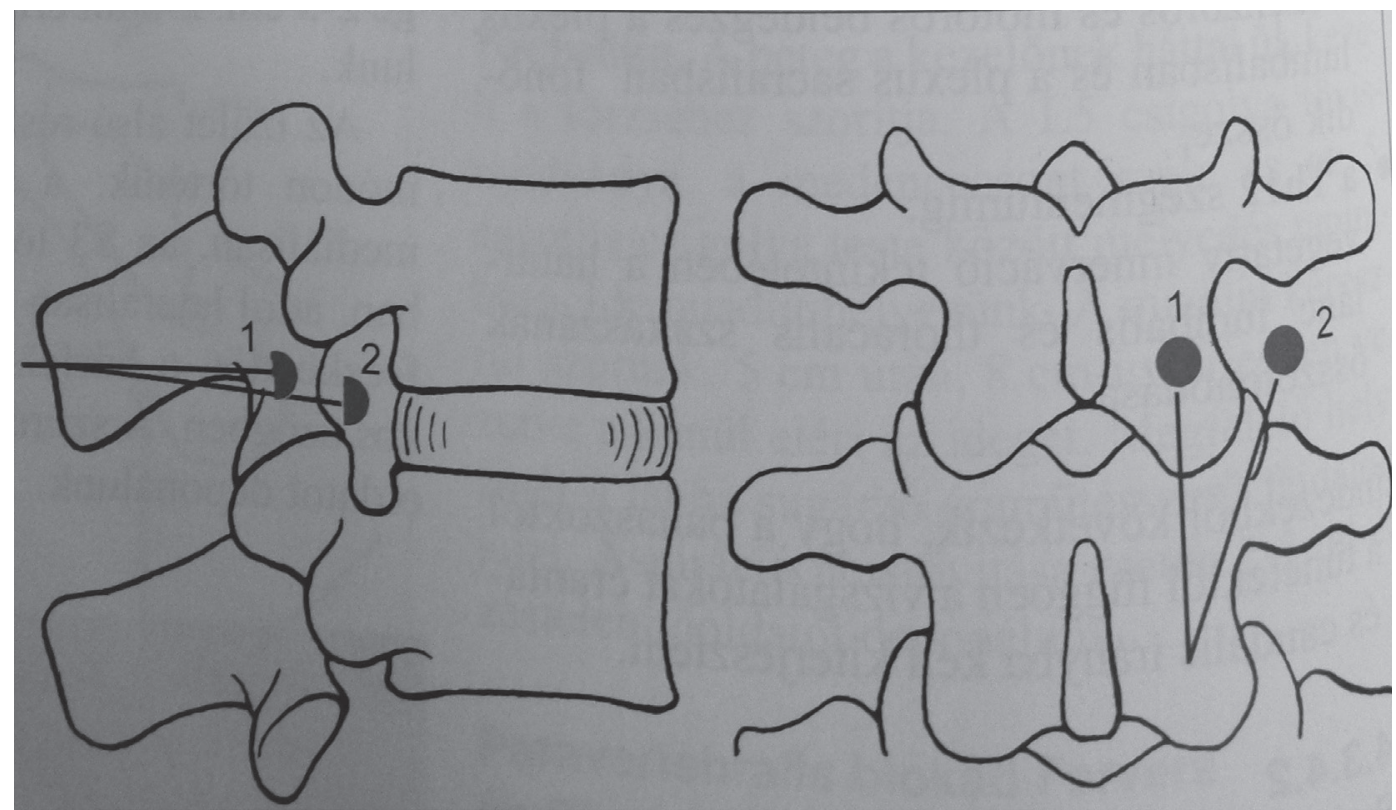

5.3. Procedure of lumbar neural therapy: 1: orientation of needle first, 2 . Deep arrival of ganglion

Fig. 5.-5-3. "Lege artis" neural therapy (according to Hunecke) in chronic, degenerative syndromes on musculoskeletal system. Neural therapy ("neuraltherapy"), therapeutic local anesthesia (TLA), or therapeutic neural blockade, are common names for the diagnostic and therapeutic use of local anesthetics (LA). In contrast to the well-defined and short-time use for analgesia in surgery, the neural therapy approach aims for long-term relief of pain and functional disorders.

(Neural therapy- A review of the therapeutic use of local anesthetics, Stefan Weinschenk a,b, $* a$ Department of Gynecological Endocrinology and Reproductive Medicine, University oj Heidelberg, Germany, b The HUNTER Group - Heidelberg University Neural Therapy Education and Research, Germany)

Questionnaire on pain and QoL was filled with all sessions during a physical examination. Visual Analog Scale (VAS), and was evaluated.

\section{RESULTS}

We recognized in N0 and N1st stage of Dupuytren's cases a significant delay of progression, moderately in N2nd. No changes were observed in progressive stages after two years' follow-up. According to delayed progression in VAS can be seen the result. (Fig. 6.) 


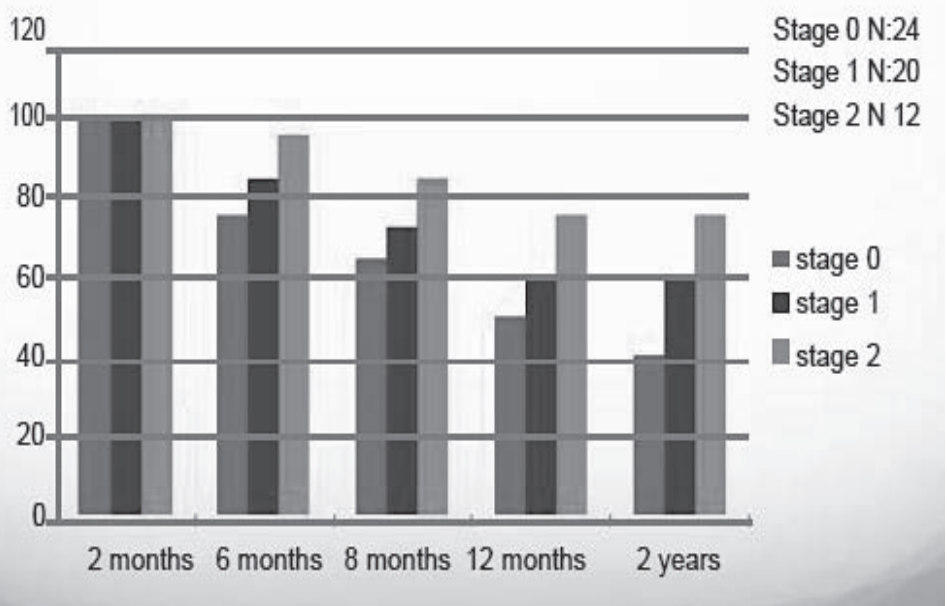

Fig. 6. Results in Dupuytran's contracture, (2013-2015).

Study of the cLBP showed significant effect of cure \& better results were achieved. We observed the most effective pain-block in cases of ischialgia, chronic lumbar disc degeneration, and cLBP in consequence of postoperative oedema. The VAS score was reduced significantly (Fig. 7.) Anecdotically we registered, that the when the regular activity was blocked due to cLBP was significantly reduced. We registered the reduce dose of analgesic pills after the treatments.

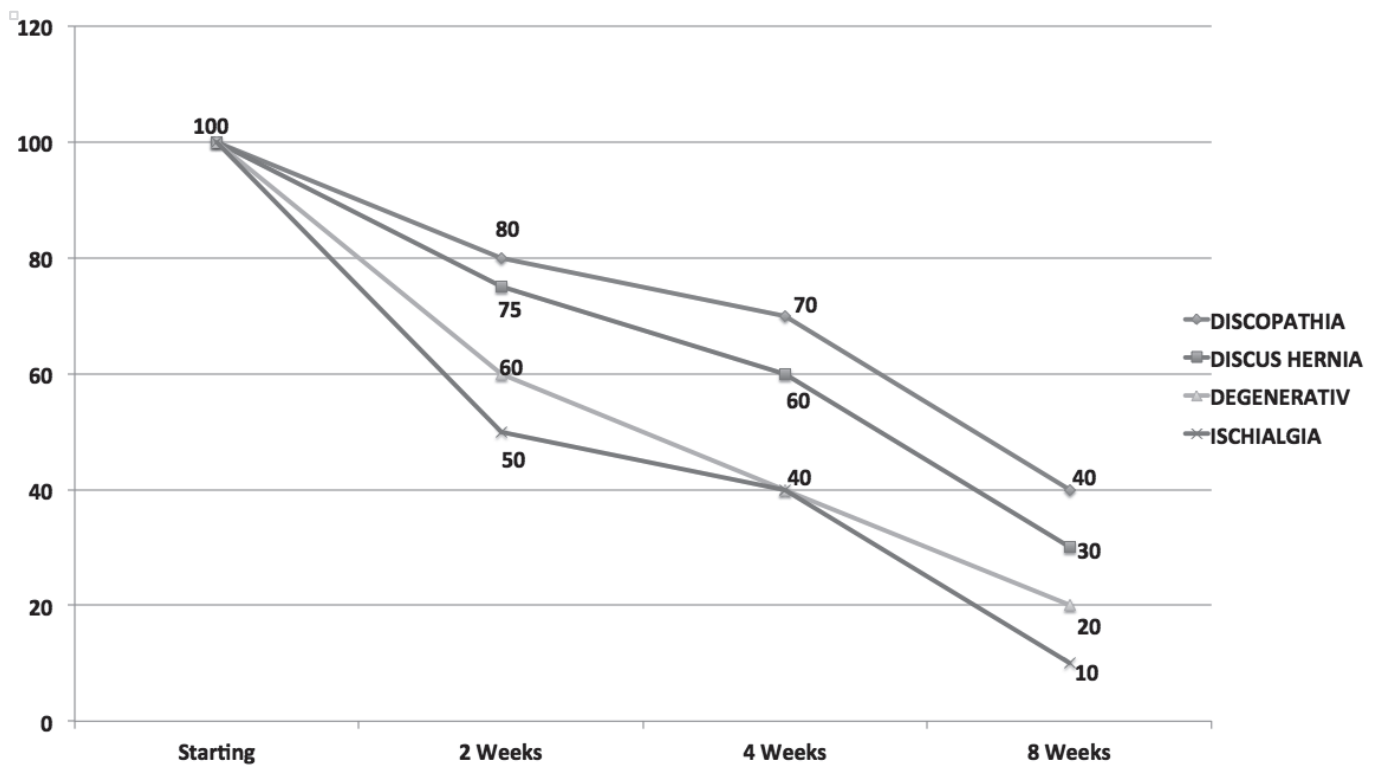

Fig. 7. Results in chronic low back pain, $(n=155)$ VAS (2013-2015) 


\section{DISCUSSION}

The targeted RF therapy was used complementary to comprehensive pharmacotherapy with the Booster device. The treatment with the device was well supported by the various medications, but importantly the pain killer analgesics were reduced gradually by time. The dominant effect of the Booster was the deep heating in the region where the electrode was positioned. The actual specific absorption rate (SAR) leads to vasodilatation in the target volume, that in turn, improves blood perfusion, and thus the drugs (and more oxygen) are transported to the treatment volume. The increased blood flow helps the higher concentration of the drug in the target as well as reduces the impedance of the drug through the walls of capillaries, and could help the cellmembrane reaction and cellular influx of the drugs, together the increased chemical reaction rate due to the elevated temperature. The improvement was expected due to the increased fibroblast activity and increased capillary angiogenesis, increased nutrient concentration and well gained metabolic activity. The synergy of the field-dependent effects [12], (optimization of membrane stimulation and activation of signal channels, etc.) is also assumed. Increased reactions to heat and field exposure (mainly the development of membrane heat shock proteins, (mHSP), which would be liberated to extracellular fluid promoting apoptosis of damaged cells [13], increased venous and lymphatic flow causing changes in the physical properties of the tissue finally. The selection of cells did not occur, but the membrane raft heating was effective as expected in nanoheating processes [2], [3]. The temperature in the targeted volume was $38-39^{\circ} \mathrm{C}$ (moderate conventional hyperthermia), which is the optimum for immune-reactions too [14].

Recognition of the distortions in the healthy tissue have some common principles and possibilities in TCM and OTM: the loss of complexity of the living organization is recognized by both methods, and the aim to reestablish the homeostatic equilibrium is common [15].

We observed, that our special permanent acupuncture method well fits to the complementary applications of treatments with Booster. The synergy of the ancient knowledge and the high-tech state-of-art of the medical knowledge could be established by this research. The positive result of this study was the recognition to apply the RF heat therapy for non-malignant, but proliferating connective tissue diseases with a positive result.

The limitation of this study is a lack of double-blind control and lack of "sham" application. We need further research in the future to clarify the exact undergoing process in selected disease group, but results show improvement and better QoL on regarded diseases. Therefore, it is advisable to use this physiotherapeutic method in the daily practice of mentioned disease after careful selection. [16,17]

\section{CONCLUSION}

OTM application is a useful, harmless additional complementary treatment for the management of selected diseases. Our objective which was giving western trained physicians clinical applications of new Oncothermia- Booster method is fulfilled. We recognized this equipment as a good physiotherapeutic complementary facility to accommodate accelerating interests in the modern complex treatment of western medicine together with TCM. 
According to our experience, the "targeted radiofrequency-physiotherapy as Booster" application is free of side effects, harmless, well-tolerable by patients, easy to apply by the skilled operation. It is advised to consider that complementary treatment for selected diseases, introducing it into daily rheumatologic practice. Also, we recognized the less intake of medicaments in cases during sessions of low back pain, which is important to avoid unnecessary side effects of medicaments (NSAID).

Abbreviations: OTH: Oncothermia, cLBP: chronic low back pain, TCM: Traditional Chinese Medicine, VAS: Visual Analog Scale, QoL: Quality of Life, DoCo: Dupuytren's contracture, RF: radiofrequency

The present scientific contribution is dedicated to the 650th anniversary of the foundation of the University of Pécs, Hungary, which is the oldest renowned University in Hungary.

\section{REFERENCES}

1. Szasz, A. Szasz, N. Szasz, O. Oncothermia: Principles and Practices, (book) SpringerVerlag. 2010.

2. Szasz, A. Electromagnetic effects in nanoscale range. Cellular response to physical stress and therapeutic application. Nova Science Publishers, Inc., 2013.

3. Szasz O, Szasz A Oncothermia - Nano-heating paradigm, Journal of Cancer Science Therapy. 6(4), 117-121, 2014.

4. Szasz A, Iluri N, Szasz O Local hyperthermia in Oncology - To Choose or not to Choose? A chapter in book: Hyperthermia, Ed: Huilgol N, ISBN 980-953-307-019-8, InTech, 2013.

5. Szasz A Challenges and solutions in oncological hyperthermia, Thermal Medicine, 29(1):123, 2013.

6. Andocs G, Szasz O, Szasz A. Oncothermia treatment of cancer: from the laboratory to clinic, Electromagnetic Biological Medicine. 28(2): 148-65, 2009.

7. Zais O, Szasz A Lyme disease and oncothermia, Conference Papers in Medicine, Vol. 2013, Article ID 275013, Hindawi Publications, 2013.

8. Hegyi G, Jian L, Szasz A Low back pain-Complex approach of treatment by different CAM modalities, Conference Papers in Medicine, Vol. 2013, Article ID 326595, Hindawi Publ. 2013.

9. Ballerini M, Baronzio GF, Capito G, Szasz O, Cassutti V, Szasz A Androtherm application for the Peyronie's disease, Conference Papers in Medicine, Vol. 2013, Article ID 962349, 2013.

10. Hegyi G. Usage of Booster (oncothermia) in rehabilitation of non-malignant conditions, 32nd Annual Conference of ICHS 2013, Panyu, China P.R. , 2013.

11. Hegyi G, Szigeti GP: Rehabilitation of Stroke Patients using Yamamoto New Scalp Acupuncture - A Pilot Study, Journal of Alternative and Complementary Medicine, Volume 18(100, pp. 1-7, 2012. 
12. Andocs G, Renner H, Balogh L, Fonyad L, Jakab C, Szasz A Strong synergy of heat and modulated electro- magnetic field in tumor cell killing, Study of HT29 xenograft tumors in a nude mice model. Strahlentherapie und Onkologie 185:120-126, 2009.

13. Yang K-L, Huang C-C, Chi M-S, Chiang H-C, Wang Y-S, Andocs G, et.al. In vitro comparison of conventional hyperthermia and modulated electro-hyperthermia, Oncotarget, doi: 10.18632/ Oncotarget. 11444, 2016.

14. Beachy, S. H.; Repasky, E. A. Toward establishment of temperature thresholds for immunological impact of heat exposure in humans. International Journal of Hyperthermia, 27, 344-352, 2011.

15. Hegyi G, Vincze Gy, Szasz A On the Dynamic Equilibrium in Homeostasis. Open Journal of Biophysics, 2:64-71, 2012.

16. Kanodia AK, Legedza ATR, Davis RB, Eisenberg DM, Phillips RS, Perceived benefit of Complementary, and Alternative Medicine (CAM) for back pain: A national survey, Journal of the American Board of Family; Medicine 23:354-36, 2010.

17. Hegyi G, Szasz O., Szasz A. Oncothermia: A New Paradigm and Promising Method in Cancer Therapies, Acupuncture \& Electro-Therapeutics Research, International Journal of Integrated Medicine 38:(3-4) pp. 161-197, 2013. 